\title{
Mediación y animación a la lectura infantil: caso mercados y plazas
}

\section{Conciliation and encouragement for child's reading in markets and squares}

\author{
Diana Priscila Saldaña Gómez ${ }^{1 *}$, César Xavier Barrera Garnica ${ }^{2}$ y Irma Jaqueline Fajardo Pacheco ${ }^{1}$ \\ ${ }^{1}$ Universidad Nacional de Educación, Ecuador \\ ${ }^{2}$ Ministerio de Educación, Ecuador \\ *diana.saldana@unae.edu.ec
}

DOI: https://doi.org/10.26871/killkana_social.v4i1.617

\begin{abstract}
Resumen
La presente investigación cualitativa abarca la temática de la lectura, dirigida a niños y niñas de 0 a 10 años, como parte de un proyecto de vinculación con la sociedad. El proceso del estudio fue la selección de la literatura infantil, la preparación de los lectores, la distribución de las zonas a intervenir y la lectura como acción principal. La finalidad de las actividades planteadas y ejecutadas es mediar, animar y promocionar la lectura a personas que se encuentren en espacios públicos como plazas y mercados, interactuando con transeúntes e hijos de comerciantes y realizando la lectura de cuentos infantiles de manera individual o grupal. Luego se ejecutaron estrategias post lectura como conversatorios, foros sobre la temática y dibujo. La metodología de investigación está centrada en la investigación acción participativa, cuyas técnicas utilizadas principalmente fueron la observación directa, las entrevistas y el registro fotográfico; todos los actores involucrados en el proceso fueron evaluados mediante diferentes instrumentos para recolectar información sobre su participación, percepción del proceso y resultados obtenidos. Luego del análisis de la información se concluye que la lectura es un recurso con múltiples beneficios escolares y colectivos que fomenta la interacción entre actores sociales, pero es un hábito poco desarrollado en el medio y nulo en espacios públicos como plazas y mercados, donde las madres de familia manifiestan que no existe ninguna estrategia de lectura realizada por ellas hacia sus hijos. Es por ello que se considera a la lectura un derecho y actividad primordial a ser insertada en la sociedad ecuatoriana mediante diferentes técnicas, donde se involucren a autoridades nacionales junto con instituciones públicas y privadas y ciudadanos voluntarios en espera de un cambio y mejora a nivel de país.
\end{abstract}

Palabras clave: lectura, plazas, mercado, vinculación, niños.

\begin{abstract}
This qualitative research covers the reading subject, aimed at children from 0 to 10 years, as part of a relationship with a society whose process was the selection of children's literature, preparation of readers, distribution of areas to intervene and reading as the main action. The purpose of the proposed and implemented activities is to mediate, encourage and promote reading to people who are found in public spaces such as squares and markets, interacting with passersby and children of merchants, and reading children's stories individually or in groups. Post-reading strategies such as conversations, forums on the subject and drawing were performed. The research methodology is focused on participatory action research. Our techniques mainly used are direct observation, interviews and photographic recording. All the actors involved in the process were evaluated through different instruments to gather information about their participation, perception of the process and results obtained. Then, the analysis of the information concludes that reading is a resource with multiple school and collective benefits that encourages interaction between social actors. However, it is a poorly developed habit in our environment and void in public spaces such as squares and markets, where mothers say that there is no reading strategy made by them towards their children. That is why it is necessary to consider reading as a fundamental right and activity to be inserted in Ecuadorian society through different techniques, involving national authorities together with public and private institutions and voluntary citizens wanting for a change and improvement at a country level.
\end{abstract}

Keywords: reading, squares market, society, children. 


\section{Introducción}

El lenguaje es un acto social, que permite la comunicación humana, a través de éste las personas pueden interactuar, expresar sus pensamientos, sentimientos e ideas, no solamente con palabras sino también con un lenguaje gestual o corporal. Lo utilizan todas las personas, todo el tiempo, no existe un límite de palabras o frases al día.

El desarrollo del lenguaje inicia desde los primeros días de nacido, con una etapa pre-lingüística que comienza con el llanto y luego pasa por varias etapas, entre sonidos fonéticos, vocálicos y continúa su desarrollo hasta la formación de palabras y frases, las cuales constituyen un recurso importante al momento de la interacción verbal con el otro. El lenguaje se desarrolla a lo largo de toda la vida de una persona, incluso en la adultez, nunca se deja de aprender una nueva palabra, un nuevo concepto e incluso el aprendizaje de un nuevo idioma. Pero ¿Qué relación tiene el lenguaje con la lectura? El tema del presente proyecto de investigación trata sobre la lectura dirigida a niños y niñas, en espacios públicos, específicamente en mercados y plazas.

La lectura en los espacios públicos es una actividad realizada por un grupo de estudiantes universitarios como parte de su proyecto de vinculación con la sociedad, dentro de un programa institucional llamado Lectura y Escritura para todos cuya finalidad es promover el desarrollo de estas actividades en diferentes espacios, buscando sensibilizar su importancia y aportar al desarrollo de infantes y desempeño de adultos. La lectura es un proceso de interpretación de grafemas mediante la vista, en la cual intervienen procesos cognitivos que permiten descifrar textos y entenderlos.

La lectura puede considerarse una actividad individual o grupal, que puede ser potenciada desde los primeros años de vida buscando estimular el lenguaje oral y escrito en niños y niñas. El proyecto espera ser mediador de lectura, el canal que conecte a los niños con los textos

podemos entender al mediador de lectura como un adulto que facilita los primeros acercamientos del niño al libro, en un proceso en el que priman la afectividad y la creación de un momento de lectura acogedor y gratificante. allí el mediador demuestra su propia dicha de leer para sembrar ese deseo en el otro, en este caso el niño y sus reducidas experiencias de lectura. (Riquelme y Munita, 2011, p.273)

Otro objetivo que se espera es realizar animación a la lectura, la cual es "una actividad que se propone el acercamiento y la profundización en los libros de una manera creativa, lúdica y placentera" (Domech citado en Chaves, 2015, p.4)

Rosenblatt (como cita Hoyos y Gallego, 2017) expresa que "todo acto de lectura es un acontecimiento o una transacción que involucra a un determinado lector y a un patrón de signos en particular, y a un texto que ocurre en un momento determinado, dentro de un contexto particular." (p.26). La actividad ejecutada en las ciudades de Cuenca y Azogues busca llevar lectura de cuentos a espacios públicos, agrupar a niños y niñas de diferentes edades, nacionalidades, cultura, condición socioeconómica, etc. buscando una mediación, animación y promoción de la lectura, al acercar a los infantes a un libro cuyo contenido es apropiado para su edad y propicia la creatividad e imaginación a través de sus historias ficticias con un toque de realidad.

\section{Marco teórico o antecedentes}

\subsection{El leer y la lectura}

Según el diccionario de la Real Academia de la Lengua, en su vigesimotercera edición (2014), leer es: "Pasar la vista por lo escrito o impreso comprendiendo la significación de los caracteres empleados", "Comprender el sentido de cualquier tipo de representación gráfica. "Entender o interpretar un texto de determinado modo", etc. Y, por lo tanto, la lectura es la acción de leer.

La lectura es una actividad social, que contiene reglas y normas, enseñada en los primeros años de la educación básica y reforzada hasta la muerte, la lectura es

un instrumento de control o un arma cultural, y también es una herramienta de la estratificación social. Pero la lectura también puede llegar a ser un obstáculo o un atajo para la ortodoxia cultural, en tanto que actividad rebelde y vagabunda. (Ramírez Leyva, 2009, p.175)

Se considera una herramienta fundamental en la educación de un país, mediante ésta, las personas viajan, conocen diferentes culturas, lugares, características, e incluso la política de un país, desde la comodidad de un espacio físico donde se encuentre leyendo.

La lectura se caracteriza por poseer varios beneficios con una sola acción, entre los cuales se puede nombrar: enriquecimiento de vocabulario, mejorar en la pronunciación, desarrollo de la memoria, aumenta la comprensión lectora, favorece la concentración, desarrolla la imaginación, entre otras. Los textos ofrecen a toda persona una variedad de vocabulario, por esa razón es muy utilizada desde tempranas edades, familiarizando a los niños la actividad de leer.

Esta acción puede darse de manera silenciosa e individual o ruidosa y grupal, en cualquier momento del día y espacio, hay personas que pueden acoplarse a cualquier espacio físico y distractores, pero otras prefieren la privacidad y el silencio. En la escuela, generalmente se la realiza en grupo, desde los primeros años de la educación básica y se utilizan textos de asignaturas de la malla curricular establecida por la autoridad máxima en educación para guiar esta actividad. Pero ¿qué pasa con el hábito lector en el país?

\subsection{Estadísticas de lectura en el país}

La república del Ecuador tiene uno de los índices de lectura más bajos de Sudamérica, un informe emitido por la CERLALC (Centro Regional para el Fomento del Libro en América Latina y el Caribe) en el año 2017, revela que los ecuatorianos leen un promedio de 0,5 libros por año mientras los chilenos leen 5,4 libros anuales. Según 
este informe de indicadores de lectura, el Ecuador posee un $43 \%$ de población lectora, frente al $92 \%$ en España o al $77 \%$ en Colombia. De ese porcentaje de lectores, el $52,2 \%$ dedica su tiempo a la lectura de libros, mientras que un $37,7 \%$ lee periódicos y un $3,7 \%$, revistas. El mismo estudio revela, que en el país aún hay preferencia por la lectura en su formato tradicional, es decir, libros, periódicos y revistas en papel y que al menos la mitad de los lectores ecuatorianos lo hacen por el gusto y el hábito de la lectura (La lectura es un hábito en construcción en el Ecuador, 2017).

Lo que se expone en estos datos estadísticos preocupan de manera general, pero sobre todo a las personas que laboran en el ámbito educativo, y es por éste motivo que la presente investigación busca desarrollar una mediación de lectura, poniendo en contacto a los niños y niñas de sectores públicos de nuestra región, con material de lectura tales como libros y argumentos de carácter infantil y científico; y una animación o motivación por la lectura del material brindado vinculando a sectores o personas que poseen poco o escaso hábito lector en nuestra sociedad.

\subsection{La lectura en la primera infancia}

Como se ha expuesto anteriormente, la lectura puede iniciar desde los primeros meses de vida, tanto en el contexto familiar como el escolar, en los centros de educación inicial o jardines infantiles, el acercamiento a la lectura generalmente es mediante cuentos, los cuales son recursos utilizados con frecuencia por las educadoras parvularias.

El cuento es un recurso que se encuentra al alcance de cualquier persona, el cual ofrece una historia secuencial, constituida por hechos reales e imaginarios, donde se destaca el papel de el o los protagonistas y su estructura generalmente está conformada por introducción, problema, desenlace y final, que en muchas ocasiones es de carácter positivo y brinda una enseñanza o un valor social. Los cuentos para niños tienen como característica, ser cortos, con pocas palabras y dibujos grandes, llamativos en su forma y contenido.

La lectura de cuento no es una estrategia aislada ni simple, puede ser apoyada de varias actividades como la dramatización, canciones, técnicas de pintura, lluvia de preguntas, entre otras; las cuales, en los infantes, tienen como fin desarrollar su lenguaje y apoyar al desarrollo integral en sus diferentes ámbitos.

Soto (2017) en su investigación El cuento como mediación pedagógica para el fortalecimiento de la lectoescritura manifiesta que

los cuentos sirvieron como recurso para fortalecer las competencias lectoras de los estudiantes, dado que afianzó de manera significativa aspectos como la fluidez en la lectura individual y la comprensión de situaciones en contexto. Adicionalmente, se mejoraron las competencias del Ser, el Saber Hacer y el Saber Ser dentro del proceso de enseñanza-aprendizaje. (p.14)
Esta actividad está muy relacionada con la educación, uno de sus principales beneficios es la adquisición del vocabulario y la enseñanza de alguna temática dentro de una asignatura, "Los libros de literatura infantil contienen tres veces más palabras de baja frecuencia (y los de preescolar dos veces más) que conversaciones de la misma extensión entre niños y adultos e incluso que conversaciones entre adultos" (Hayes y Ahrens citado en Goikoetxea y Martínez, 2015, p.9). Al considerarse a la lectura como un acto social que enriquece el conocimiento podría ser utilizada sin límites en el contexto educativo, pero también puede ser llevada a contextos no formales o no convencionales.

\subsection{Lectura y su vinculación con la sociedad}

Luego de señalar varios beneficios de la lectura, la investigación se ha preguntado ¿por qué no llevar la lectura hacia la sociedad?, en cantones, parroquias, barrios y sobre todo en lugares poco comunes donde se considera que la lectura no es una actividad frecuente, como lo son, los parques, las calles o locales comerciales.

En muchos estudios o investigaciones se puede encontrar el término lectura compartida, el cual Goikoetxea y Martínez (2015) lo considera como "una de las actividades más importantes que los adultos pueden realizar con niños en edad preescolar por los beneficios que tiene en el desarrollo del lenguaje" (p.6). La palabra compartida abarca la interacción entre dos o más personas y como lo mencionan los autores, en la primera infancia la lectura está a cargo de una persona adulta quien presenta un texto, anima a la lectura y se constituye como un canal o medio para promocionarla.

La lectura compartida constituye un beneficio para todos los participantes de la misma, el que lee mejora destrezas lectoras y de comprensión y el que escucha lo realiza de manera activa, adquiriendo mayor vocabulario, "numerosos estudios demuestran que la lectura interactiva (que incluye etiquetar, señalar, preguntar, participar en diálogos mientras se lee) es más efectiva en el aprendizaje de palabras nuevas" Blewitt, Rump, Shealy y Cook (citado en Goikoetxea y Martínez, 2015, p.13). y todas las actividades enunciadas anteriormente se realizan con frecuencia luego de una lectura. En los infantes se obtiene una reacción espontánea, en la cual no solamente se desarrollan habilidades cognitivas sino también emocionales, al decir lo que piensan o sienten con respecto al texto, mientras el adulto interacciona y retroalimenta las participaciones de las personas involucradas.

Un gran ejemplo de vinculación con la sociedad desde la lectura, en épocas pasadas, fue la creación de bibliotecas barriales o populares en el año 1879 en varias comunidades de Argentina, luego de la crisis económica y política del 2001. Donde se creó pequeñas bibliotecas con personas del sector y solicitudes de donaciones, con la finalidad de servir al barrio, promoviendo un derecho público de los ciudadanos, a las bibliotecas las consideraban como "una 
herramienta estratégica para ejercer la democracia directa y la política solidaria” (Schwartz, 2017, p.19).

\subsection{La comprensión lectora}

El presente trabajo pretende y busca la mediación y la animación a la lectura, pero no deja de lado la importancia de propiciar una comprensión lectora adecuada, en los participantes, objeto de esta investigación, entendiéndose entonces por comprensión lectora como aquel fenómeno que provoca que los lectores analicen, reflexionen e interpreten una larga lista de ideas presentes en un grupo de palabras, un párrafo o un texto literario, tratando de relacionar con los conocimientos preexistentes, generando y provocando situaciones de desaprendizaje y nuevos aprendizajes que serán de beneficio para su desarrollo cognitivo e intelectual.

Del Ángel y Rodríguez (2007) hacen referencia a la evaluación estudiantil internacional PISA y se centran en el área de lectura, donde manifiestan que su valoración se encuentra "en función de tres escalas: a) obtención de información, b) interpretación de textos, y c) reflexión y evaluación; los niveles de competencia tienen como objetivo clasificar el desempeño de los estudiantes y describir lo que son capaces de hacer" (p.18). Según el puntaje obtenido, el estudiante se sitúa en uno de los 5 niveles detallados a continuación:

Tabla 1: Niveles de evaluación PISA en el área de competencia de lectura

\begin{tabular}{|c|c|}
\hline Niveles & Características \\
\hline $\begin{array}{l}\text { Nivel } 5 \text { (más } \\
\text { de } 625 \text { puntos) }\end{array}$ & $\begin{array}{l}\text { Los estudiantes son capaces de completar reactivos de } \\
\text { lectura sofisticados }\end{array}$ \\
\hline $\begin{array}{l}\text { Nivel } 4 \text { (de } 553 \\
\text { a } 625 \text { puntos) }\end{array}$ & $\begin{array}{l}\text { Los estudiantes son capaces de responder reactivos de } \\
\text { lectura dificiles }\end{array}$ \\
\hline $\begin{array}{l}\text { Nivel } 3 \text { (de } 481 \\
\text { a } 552 \text { puntos) } \\
\end{array}$ & $\begin{array}{l}\text { Los estudiantes son capaces de manipular reactivos de } \\
\text { lectura de complejidad moderada }\end{array}$ \\
\hline $\begin{array}{c}\text { Nivel } 2 \text { (de } 408 \\
\text { a } 480 \text { puntos) }\end{array}$ & $\begin{array}{l}\text { Los estudiantes son capaces de responder reactivos básicos } \\
\text { de lectura }\end{array}$ \\
\hline $\begin{array}{l}\text { Nivel } 1 \text { (de } 335 \\
\text { a } 407 \text { puntos) }\end{array}$ & $\begin{array}{l}\text { Los estudiantes son capaces de responder correctamente } \\
\text { sólo los reactivos de lectura menos complejos como ubicar } \\
\text { un fragmento de información, identificar el tema principal } \\
\text { de un texto o establecer una conexión sencilla con el cono- } \\
\text { cimiento cotidiano. }\end{array}$ \\
\hline
\end{tabular}

Fuente: Minerva del Ángel - Adolfo Rodríguez

Los autores también indican que los estudiantes con una valoración por debajo de 335 puntos no son capaces de realizar una lectura básica, pueden pronunciar las palabras y leer técnicamente, pero se evidencia una escasa comprensión, lo cual repercutirá en su vida, sobre todo en el ámbito escolar, en el cual se considera a la lectura como una herramienta básica para cualquier asignatura.

\section{Metodología}

La investigación realizada con enfoque cualitativo utilizó como principal método la investigación acción participativa, en la cual estudiantes, en grupos, recorrían espacios públicos llevando lectura a personas de toda edad, pero en mayor cantidad a niños y niñas de 0 a 10 años, recorriendo la zona urbana de Cuenca y Azogues, acercándose a las personas que encontraban en las calles, plazas, mercados y locales comerciales, invitándolos a sentarse en bancas, veredas, juegos infantiles o espacios verdes para introducir la lectura con un saludo y explicación de la actividad, desarrollando empatía, generalmente formaban grupos de 2 a 6 niños y niñas, ya que en ocasiones si uno participaba en la actividad, más personas se acercaban por curiosidad y se quedaban escuchando la lectura.

Al finalizar las lecturas las estudiantes realizaban preguntas a los niños sobre la misma, repasan nuevamente el texto, acercan al niño al material físico o realizan alguna actividad que los infantes les solicitan, como cantar o pintar.

Un mes posterior a la finalización de las actividades se realizó encuestas a las madres de familia y niños del mercado, para identificar el impacto que tuvo el proyecto sobre los actores principales y sus familias, las encuestas fueron sistematizada y analizadas para el detalle de los resultados. El registro fotográfico fue otro instrumento que se consideró para analizar los resultados e identificar a los actores atendidos, junto con la matriz de seguimiento geográfica que fue sistematizada para identificar los lugares recorridos.

\section{Resultados}

Posterior a la ejecución de las actividades de lectura se realizó un levantamiento de información sobre la percepción de los actores. La primera acción que se desarrolló fueron entrevistas a las madres de familia de los mercados intervenidos, las cuales manifestaron su agrado con las actividades realizadas y comentaron sobre la permanencia de los niños en su trabajo, los cuales generalmente pasan en sus puestos (locales comerciales pequeños) realizando tareas escolares, jugando en el celular, viendo la TV o jugando dentro del mercado con hijos de otras comerciantes. Al preguntar sobre la lectura en casa o en ese espacio, expresaron que no existe lectura por parte de ellas hacia sus hijos en ningún momento ni espacio, la respuesta fue similar en todos los casos con la confirmación de nula lectura por parte de madres a hijos.

Al conversar con los niños sobre la actividad, manifestaron que les gustó realizarla, les llamó la atención el grupo de personas que asistieron como lectores, por su apariencia colorida y su amabilidad, además declararon que no solamente escucharon la lectura sino pasaron tiempo con ellas realizando actividades de dibujo y conversando sobre los personajes y escenas del cuento.

Al consultar si desearían que estas actividades se repitan, el total de beneficiarios respondió de manera afirmativa, lo cual indica, a través de sus palabras y gestos, que la lectura llevada a estos espacios se considera no solamente educativa sino recreativa ante los niños y sus madres, pudiendo realizarla con mayor frecuencia o duración, ya que aparte de ser el mediador entre el niño y el texto; el lector, mediante su actitud positiva y empatía, se convierte en una 
persona que genera alegría con las estrategias utilizadas post lectura y presentadas a manera de juego.

Durante la ejecución del proyecto las personas lectoras registraban sus visitas a los diferentes lugares visitados, las plazas, parques, calles y mercados y documentaron sus actividades de manera fotográfica, la misma que sirvió para analizar lugares recorridos, identificar edades y género de las personas, pero sobre todo lo que se pudo identificar fue la expresión facial y corporal de todos los participantes, se observa rostros de emoción y alegría, grupos de niños sentados en el piso, en la vereda o en el llano mirando el cuento con atención, niños en brazos interviniendo en las actividades, lectoras sentadas en juegos infantiles o puestos de venta de alimentos, junto con niños e incluso perros, leyendo.

Las lectoras indicaron haberse sentido cansadas pero contentas al realizar las actividades, cansadas por el trabajo en las calles, el caminar por varias horas, independientemente del estado del tiempo, pero alegres al ver la reacción de los niños, el asombro y curiosidad ante los cuentos y la participación activa y voluntaria, de igual manera sintieron el apoyo de las madres de familia, quienes a lo lejos observaban discretamente la actividad.

\section{Conclusiones}

Una vez realizada la presente investigación, se obtienen las siguientes conclusiones:

Existe un bajo interés por la lectura en nuestro país, más aún en lugares de conglomeración masiva como lo son los mercados de las principales ciudades de la región.

Los individuos objeto de estudio, despiertan gran interés por el hábito lector, lo que se manifiesta por su actitud y comportamiento frente a los jóvenes que participan como mediadores y animadores.

Las personas adultas, madres de familia que laboran en el mercado, no poseen hábitos de lectura tanto para su autopreparación como para ayudar a sus hijos con las tareas escolares, sin embargo, manifiestan una profunda empatía y simpatía con las actividades desarrolladas.

Las jóvenes estudiantes universitarias adquieren experiencia y gusto por el servicio comunitario prestado.

\section{Recomendaciones}

Continuar con actividades de lectura en espacios no convencionales. Planificar y ejecutar estrategias de fomento a la lectura. Realizar campañas de lectura a nivel parroquial, cantonal o zonal. Documentar los procesos de lectura para motivar a la sociedad. Apoyar a los proyectos o acciones de vinculación con la sociedad. Realizando investigaciones con características similares.

\section{Referencias Bibliográficas}

Del Ángel, M., y Rodríguez, A. (2007). La promoción de la lectura en méxico. Infodiversidad, 11, 11-40.

La lectura es un hábito en construcción en el Ecuador. (2017, abril). Descargado de https://www.elcomercio.com/ tendencias/lectura-ecuador-libro -habitos-cultura.html

Ramírez Leyva, E. M. (2009). ¿ qué es leer?¿ qué es la lectura? Investigación bibliotecológica, 23(47), 161188.

Reatiga, M. S. (2017). El cuento como mediación pedagógica para el fortalecimiento de la lectoescritura. Zona próxima: revista del Instituto de Estudios Superiores en Educación(27), 51-65.

Riquelme, E., y Munita, F. (2011). La lectura mediada de literatura infantil como herramienta para la alfabetización emocional. Estudios pedagógicos (Valdivia), 37(1), 269-277.

Schwartz, M. (2017). Cacerolazos y bibliotecas: lectura solidaridad y espacio público después de la crisis argentina de 2001-2002. Revista de Humanidades(35), $15-42$.

Recibido: 5 de septiembre de 2019

Aceptado: 6 de marzo de 2020 
